\title{
Modeling photon output caused by backscattered radiation into the monitor chamber from collimator jaws using a Monte Carlo technique
}

\author{
H. Helen Liua) \\ Department of Radiation Physics, The University of Texas M. D. Anderson Cancer Center, Houston, \\ Texas 77030 \\ T. Rockwell Mackie \\ Department of Medical Physics, University of Wisconsin, Madison, Wisconsin 53706
}

Edwin C. McCullough

Division of Radiation Oncology, Mayo Clinic and Foundation, Rochester, Minnesota 55905

(Received 25 October 1999; accepted for publication 11 January 2000)

\begin{abstract}
Dose per monitor unit in photon fields generated by clinical linear accelerators can be affected by the backscattered radiation into the monitor chamber from collimator jaws. Thus, it is necessary to account for the backscattered radiation in computing monitor unit setting for a treatment field. In this work, we investigated effects of the backscatter from collimator jaws based on Monte Carlo simulations of a clinical linear accelerator. The backscattered radiation scored within the monitor chamber was identified as originating either from the upper jaws ( $Y$ jaws), or from the lower jaws $(X$ jaws). From the results of Monte Carlo simulations, ratios of the monitor-chamber-scored dose caused by the backscatter to the dose caused by the forward radiation, $R(x, y)$, were modeled as functions of the individual $X$ and $Y$ jaw positions. The amount of the backscattered radiation for any field setting was then computed as a compound contribution from both the $X$ and $Y$ jaws. The dose ratios of $R(x, y)$ were then used to calculate the change in photon output caused by the backscatter, $S_{c b}(x, y)$. Results of these calculations were compared with available measured data based on counting the electron pulses or charge from the electron target of an accelerator. Data from this study showed that the backscattered radiation contributes approximately $3 \%$ to the monitorchamber-scored dose. A majority of the backscattered radiation comes from the upper jaws, which are located closer to the monitor chamber. The amount of the backscatter decreases approximately in a linear fashion with the jaw opening. This results in about a $2 \%$ increase of photon output from a $10 \mathrm{~cm} \times 10 \mathrm{~cm}$ field to a $40 \mathrm{~cm} \times 40 \mathrm{~cm}$ field. The off-axis location of the jaw opening does not have a significant effect on the magnitude of the backscatter. The backscatter effect is significant for monitor chambers using kapton windows, particularly for treatment fields using moving jaws. Applying the backscatter correction improves the accuracy of monitor-unit calculation using a model-based dose calculation algorithm such as the convolution method. (C) 2000 American Association of Physicists in Medicine. [S0094-2405(00)00904-4]
\end{abstract}

Key words: photon output factor, Monte Carlo simulation, backscattered radiation, monitor chamber, convolution dose calculation

\section{INTRODUCTION}

The variation of photon output with field geometries has been studied extensively in the past. This is an issue of concern because in conventional approaches, output factors are used directly in computing monitor units for photon treatment fields. In particular, the use of new treatment techniques such as dynamic wedges and intensity modulated radiation therapy using dynamic jaw movement requires that the photon output be predicted with a high degree of accuracy for individual component fields. This warrants meticulous investigation of the causes of the changing photon output with field collimation.

In a previous work, ${ }^{1}$ we showed that the change of photon output as a function of field geometry is essentially caused by three factors: the head scatter, the phantom scatter, and the backscattered radiation from collimator jaws into monitor chambers. We developed a convolution/superposition algorithm to compute the photon output or dose per monitor unit. This algorithm uses a dual-source photon beam model derived from Monte Carlo simulation of clinical linear accelerators. ${ }^{2}$ Because photon energy fluence output from linear accelerators can be modeled directly using Monte Carlo simulations, we were able to accurately predict the changes of the head scatter and phantom scatter for different photon fields.

Our previous study showed that the backscattered radiation increased the photon output by about $2 \%-3 \%$ from a $3 \mathrm{~cm} \times 3 \mathrm{~cm}$ field to a $40 \mathrm{~cm} \times 40 \mathrm{~cm}$ field on Varian 2100 accelerators. The change of the backscatter depends on the accelerator structure and the monitor chamber construction, as discussed by other research groups as well. ${ }^{3-11}$ Although the effect of the backscatter is small compared to that of the head scatter and phantom scatter, to achieve more accurate 
dose calculation, it is important to include the correction for the backscatter in using the convolution algorithm or any other model-based methods. ${ }^{12}$ However, how to determine the effect of the backscatter poses a challenging problem, because the change in photon output caused by the backscatter alone is often shadowed, and thus, difficult to assess experimentally. In the past, a number of methods were investigated, including those using ionization chambers and phantoms in telescopic settings, ${ }^{3-7}$ as well as techniques based on measuring electron pulses, current, or charge from the electron target of linear accelerators. ${ }^{5,8-11}$ In general, these published data were limited to symmetrical field settings used for the measurements. Thus, it remains unknown how to extrapolate the available data and be able to predict the effect of the backscatter for any arbitrary field.

In the current study, we applied a Monte Carlo technique to simulate linear accelerators and to study the backscatter from collimator jaws to the monitor chamber. Recently, application of Monte Carlo simulation has experienced tremendous growth, particularly in studying clinical accelerators. ${ }^{13-16}$ This is because Monte Carlo simulation enables us to study the accelerator at a greater depth in identifying individual sources of the backscatter from collimator jaws. The motivation for this work is to model the backscattered radiation as functions of the upper $(Y)$ and lower $(X)$ jaw positions. These data are not available currently except for symmetric fields. Therefore, the amount of the backscattered radiation and the resulting photon output can be calculated for any jaw opening, including asymmetric fields and fields using dynamic jaw movement, such as dynamic wedges or intensity-modulated beams. As a result, the total photon output and monitor units can be calculated more accurately for these fields with correction for the backscatter.

\section{MATERIALS AND METHODS}

Varian Clinac 2100C linear accelerators generating 10 $\mathrm{mV}$ photon beams (Varian Oncology Systems, Palo Alto, CA) were simulated using the Monte Carlo code BEAM ${ }^{13}$ (1998 version). The simulation of the accelerator was accomplished in two stages. The first stage started from an electron target, and included a primary collimator and a flattening filter. The phase space data generated from this stage was universal for all the jaw settings, and thus, were used as the input for the rest of the simulation. The second stage of the simulation involved a monitor chamber, a secondary collimator for the accelerator head shielding, and two pairs of jaws [Fig. 1(a)]. The monitor chamber was simulated using the BEAM component module "Chamber" [Fig. 1(b)]. The dose-scoring regions of the monitor chamber were simulated as two slabs of air sandwiched by three layers of kapton separation windows. The central dose regions are surrounded by the chamber wall, chamber gap, and container wall. The monitor chamber is in fact made of two parallel plate chambers denoted "MU1" and "MU2" in Fig. 1(b). Information about the geometries and composition of the monitor chamber used in the simulation was obtained from the accelerator manufacturer and from actual measurements.
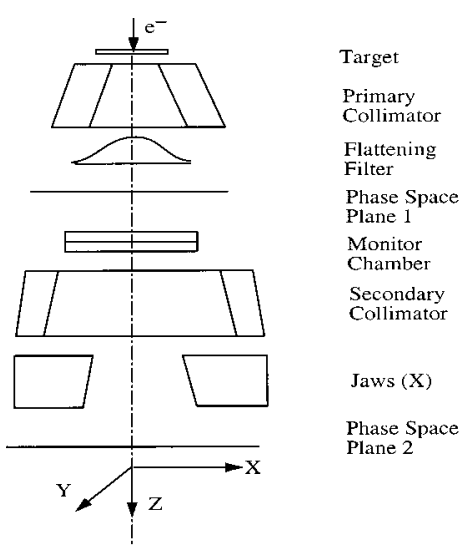

Monitor Chamber

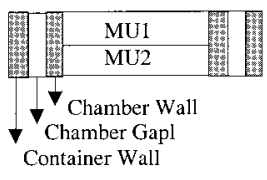

(a)

(b)

FIG. 1. Schematic drawing of the component modules of a linear accelerator used in the Monte Carlo simulation. (a) Linear accelerator; (b) monitor chamber.

The parameters used for the EGS4 simulation were: AE $=0.700 \mathrm{MeV}, \quad \mathrm{AP}=0.01 \mathrm{MeV}, \quad$ ECUT $=0.611 \mathrm{MeV}$, and PCUT $=0.1 \mathrm{MeV}$. The higher ECUT and PCUT values were used to improve the efficiency of the simulations. The results were verified against those using lower ECUT and PCUT of 0.511 and $0.01 \mathrm{MeV}$ to assure simulation accuracy. The default PRESTA electron transport algorithm from BEAM ${ }^{13}$ was used in the simulation. The variance-reduction technique of "photon forcing", 13 was used within the monitor chamber dose-scoring regions to increase the probability of photon interactions within the air slabs. The "electron range rejection" technique ${ }^{13}$ was turned off during the second stage of the simulation to achieve higher accuracy of dose scoring. Other technical details of the simulation have been reported in a previous work. ${ }^{2}$

The particles reaching the monitor chamber and contributing to dose deposition were tagged using the "latch" feature of the BEAM code. ${ }^{13}$ Therefore, the monitor-chamberscored dose was separated into three components according to the origins of the particles reaching the chamber. These components are: dose from the forward particles alone, doses from particles that are either originated or scattered from the $X$ jaws, or the $Y$ jaws. The latter two components were used to identify the backscattered dose in the chamber. Particles that happened to interact with both the $X$ and $Y$ jaws were included in the dose component from the $X$ jaws only. To study the variation of the backscatter with different jaw positions, the exact same particles output from the first stage of the simulation were input to the second stage of the simulation. The number of the phase space particles used in the second stage of the simulation was 50 million. The resulting standard errors of the total monitor-chamber-scored dose and the maximum dose caused by the backscatter were approximately $0.5 \%$ and $2.5 \%$, respectively.

The following jaw settings were used in the second stage of the simulation (all positions are specified at the isocenter plane).

(1) The $Y_{2}$ jaw was parked at $20 \mathrm{~cm}$ off axis. The $Y_{1}$ jaw was moved across the field until it was fully closed with the $Y_{2}$ jaw. The $X$ jaws were completely opened. This setting 
was used to study the backscatter as a function of the $Y$ jaw position.

(2) The $Y$ jaws were completely opened $40 \mathrm{~cm}$ apart. The $X_{2}$ jaw was parked at $20 \mathrm{~cm}$ off axis, whereas the $X_{1}$ jaw was moved across the field until it was fully closed with the $X_{2}$ jaw. This setting was used to study the backscatter as a function of the $X$ jaw position without the $Y$ jaw blocking.

(3) The $X$ jaws were completely closed. The $Y_{2}$ jaw was parked at $20 \mathrm{~cm}$ off axis, whereas the $Y_{1}$ jaw was moved across the field until it was fully closed with the $Y_{1}$ jaw. This setting was used to study the effect of the $Y$ jaw blocking on the backscatter from the $X$ jaws.

From simulating a field with the $X$ and $Y$ jaw setting of $\left(x_{1}, x_{2}, y_{1}, y_{2}\right)$, we obtained the monitor-chamber-scored dose from the forward radiation alone, $D_{f}\left(x_{1}, x_{2}, y_{1}, y_{2}\right)$, and dose from the backscattered radiation $D_{b}\left(x_{1}, x_{2}, x_{1}, y_{2}\right)$. Because the top chamber "MU1' is used to terminate radiation beams on the Varian machines, we used the dose scored within the "MU1" for all the following calculations. Because the exact same phase space particles were input for the second stage of the simulations, $D_{f}\left(x_{1}, x_{2}, y_{1}, y_{2}\right)$ was used to normalize $D_{b}\left(x_{1}, x_{2}, y_{1}, y_{2}\right)$ in obtaining the relative backscatter $R\left(x_{1}, x_{2}, y_{1}, y_{2}\right)$, i.e.,

$$
R\left(x_{1}, x_{2}, y_{1}, y_{2}\right)=D_{b}\left(x_{1}, x_{2}, y_{1}, y_{2}\right) / D_{f}\left(x_{1}, x_{2}, y_{1}, y_{2}\right) \text {. }
$$

Here, the monitor-chamber-scored dose rather than the energy fluence was used to quantify the relative backscatter because the dose or energy deposition reflects the charge collected by the monitor chamber more realistically than the energy fluence. This is due to the fact that the backscattered radiation is composed of low energy photons and electrons, whose absorption coefficient and stopping power change rapidly with decreasing energy.

By simulating fields with the same $Y$ jaw openings but different $X$ jaw openings, we verified that the backscatter of the $Y$ jaws was independent of the $X$ jaw scatter. Therefore, $R\left(x_{1}, x_{2}, y_{1}, y_{2}\right)$ is a sum of the relative backscatter from the $X$ and $Y$ jaws, $R_{x}\left(x_{1}, x_{2}, y_{1}, y_{2}\right)$ and $R_{y}\left(y_{1}, y_{2}\right)$, respectively, i.e.,

$$
R\left(x_{1}, x_{2}, y_{1}, y_{2}\right)=R_{x}\left(x_{1}, x_{2}, y_{1}, y_{2}\right)+R_{y}\left(y_{1}, y_{2}\right) .
$$

The relative backscatter from the $X$ jaws is dependent on both the $X$ and $Y$ jaw positions, whereas the scatter from the $Y$ jaws is only dependent on the $Y$ jaw opening. Furthermore, the backscatter from the $Y_{1}$ and $Y_{2}$ jaws, $R_{y_{1}}\left(y_{1}\right)$ and $R_{y_{2}}\left(y_{2}\right)$, can be considered relatively independent of each other, especially when the jaws are far apart. This is because only a small fraction of the particles can be scattered to both pieces of the $Y$ jaws and backscattered to the monitor chamber. Thus, $R_{y}\left(y_{1}, y_{2}\right)$ was computed as

$$
R_{y}\left(y_{1}, y_{2}\right)=R_{y_{1}}\left(y_{1}\right)+R_{y_{2}}\left(y_{2}\right) \text {. }
$$

From simulations of the fields with the first series of jaw settings, we obtained $R_{y}\left(y_{1}, y_{2}=20\right)$ with the $Y_{1}$ jaw moved across the field and the $Y_{2}$ jaw parked aside. Assuming $R_{y_{1}}\left(y_{1}=20\right)$ is equal to $R_{y_{2}}\left(y_{2}=20\right)$, which is half of
$R_{y}\left(y_{1}=20, y_{2}=20\right)$, we calculated $R_{y_{1}}\left(y_{1}\right)$ as

$$
R_{y_{1}}\left(y_{1}\right)=R_{y}\left(y_{1}, y_{2}=20\right)-R_{y}\left(y_{1}=20, y_{2}=20\right) / 2 \text {. }
$$

Due to symmetry, this $R_{y_{1}}\left(y_{1}\right)$ is the same with $R_{y_{2}}\left(y_{2}\right)$ obtained with the $Y_{2}$ jaw moved across the field and the $Y_{1}$ jaw parked aside. Thus, $R_{y_{1}}\left(y_{1}\right)$ in fact gives the backscattered dose from a single piece of the $Y$ jaws as a function of its position. Thus, for any $Y$ jaw setting $\left(y_{1}, y_{2}\right), R_{y}\left(y_{1}, y_{2}\right)$ was computed based on Eq. (3) using the available data of $R_{y_{1}}\left(y_{1}\right)$.

The computation of $R_{x}\left(x_{1}, x_{2}, y_{1}, y_{2}\right)$ is more complicated because the area of the $X$ jaws that is visible to the monitor chamber depends on both the $X$ and $Y$ jaw openings. To quantify the backscatter from the $X$ jaws, we defined another term $P_{y}\left(y_{1}, y_{2}\right)$, which describes the relative amount of the $X$ jaw backscatter that can pass through the $Y$ jaw opening, i.e.,

$$
R_{x}\left(x_{1}, x_{2}, y_{1}, y_{2}\right)=R_{x}\left(x_{1}, x_{2}, y_{1}=y_{2}=20\right) P_{y}\left(y_{1}, y_{2}\right),
$$

in which $R_{x}\left(x_{1}, x_{2}, y_{1}=y_{2}=20\right)$ is the backscatter from the $X$ jaws with the fully opened $Y$ jaws. Similar to the approach used for $R_{y}\left(y_{1}, y_{2}\right)$ [Eq. (3)], $R_{x}\left(x_{1}, x_{2}, y_{1}=y_{2}=20\right)$ is calculated from the individual components of the $X_{1}$ and $X_{2}$ jaws, i.e.,

$$
\begin{aligned}
& R_{x}\left(x_{1}, x_{2}, y_{1}=y_{2}=20\right) \\
& \quad=R_{x_{1}}\left(x_{1}, y_{1}=y_{2}=20\right)+R_{x_{2}}\left(x_{2}, y_{1}=y_{2}=20\right) .
\end{aligned}
$$

Data of $R_{x_{1}}\left(x_{1}, y_{1}=y_{2}=20\right)$ and $R_{x_{2}}\left(x_{2}, y_{1}=y_{2}=20\right)$ were obtained by moving the $X_{1}$ jaw across the field while the $Y$ jaws were fully opened (the second series of jaw settings used). The method is the same as that used in calculating $R_{y_{1}}\left(y_{1}\right)$ as shown by Eq. (4).

To account for the blocking effect caused by both the $Y_{1}$ and $Y_{2}$ jaws, we modeled $P_{y}\left(y_{1}, y_{2}\right)$ as

$$
P_{y}\left(y_{1}, y_{2}\right)=P_{y_{1}}\left(y_{1}\right)+P_{y_{2}}\left(y_{2}\right)
$$

in which $P_{y_{1}}\left(y_{1}\right)$ or $P_{y_{2}}\left(y_{2}\right)$ is the relative contribution of a single piece of the $Y$ jaws in passing the $X$ jaw backscatter. $P_{y}\left(y_{1}, y_{2}\right)$ has a maximum of unity with the fully opened $Y$ jaws, and a minimum of zero with a fully closed $Y$ jaws, i.e.,

$P_{y}\left(y_{1}=20, y_{2}=20\right)=P_{y_{1}}\left(y_{1}=20\right)+P_{y_{2}}\left(y_{2}=20\right)=1.0$,

$P_{y}\left(y_{1}=-20, y_{2}=20\right)=P_{y_{1}}\left(y_{1}=-20\right)+P_{y_{2}}\left(y_{2}=20\right)=0.0$.

Here a negative $Y_{1}$ or $Y_{2}$ jaw position indicates that the jaw passes the central axis and approaches the opposing jaw. The resulting $P_{y_{1}}\left(y_{1}\right)$ or $P_{y_{2}}\left(y_{2}\right)$ has a maximum value of 0.5 when the jaw is fully opened $20 \mathrm{~cm}$ off axis $\left(y_{1}\right.$ or $y_{2}$ $=20$ ), and a minimum value of -0.5 when the jaw passes the central axis by $20 \mathrm{~cm}\left(y_{1}\right.$ or $\left.y_{2}=-20\right)$. Hypothetically, this means that each piece of the $Y$ jaws can allow a maximum of $50 \%$ for the $X$ jaw backscatter to pass through; when 
the $Y_{1}$ or $Y_{2}$ jaw moves across the central axis, it reduces the amount of the $X$ jaw backscatter by "deducting" the contribution of the opposing jaw.

To determine $P_{y_{1}}\left(y_{1}\right)$ or $P_{y_{2}}\left(y_{2}\right)$, we closed the $X$ jaws completely to allow for the maximum backscatter from the $X$ jaws, and moved the $Y$ jaws sequentially, as explained earlier for the third series of jaw settings used. From the results of the simulation, $P_{y}\left(y_{1}, y_{2}=20\right)$ was calculated using the definition of Eq. (5). Similar to the approach used in calculating $R_{y_{1}}\left(y_{1}\right)$ and $R_{x_{1}}\left(x_{1}\right), P_{y_{1}}\left(y_{1}\right)$ was then calculated as

$$
P_{y_{1}}\left(y_{1}\right)=P_{y}\left(y_{1}, y_{2}=20\right)-P_{y_{2}}\left(y_{2}=20\right)
$$

with $P_{y_{2}}\left(y_{2}=20\right)$ equal to 0.5 . In this approach, the fully closed $X$ jaw was used to model the $P_{y}$ function and was used subsequently to compute $P_{y}$ for other $X$ jaw settings. The reason for using the fully closed $X$ jaw is to obtain the maximum $X$ jaw backscatter, thus minimizing the statistical uncertainty in calculating $P_{y_{1}}\left(y_{1}\right)$. We also assumed that the $P_{y}$ function is independent of the $X$ jaw opening. This assumption will be addressed in Sec. III.

Notice also that if both the $Y$ jaws are fully closed with the $Y_{1}$ and $Y_{2}$ jaws parked at a position $y_{i}, P_{y}\left(y_{1}, y_{2}\right)$ should become zero, i.e.,

$$
P_{y}\left(y_{1}, y_{2}\right)=P_{y_{1}}\left(y_{1}=-y_{i}\right)+P_{y_{2}}\left(y_{2}=y_{i}\right)=0 .
$$

Thus,

$$
P_{y_{1}}\left(y_{1}=-y_{i}\right)=-P_{y_{2}}\left(y_{2}=y_{i}\right)=-P_{y_{1}}\left(y_{1}=y_{i}\right) .
$$

This means that $P_{y_{1}}\left(y_{1}\right)$ should also be symmetric against the central axis with $P_{y_{1}}\left(y_{1}=0\right)=P_{y_{2}}\left(y_{2}=0\right)=0$. This feature was used later to fit the data of $P_{y_{1}}\left(y_{1}\right)$ as discussed in Sec. III.

Upon the calculation of $R\left(x_{1}, x_{2}, y_{1}, y_{2}\right)$ or $R(x, y)$ for simplification, the change of photon output caused by the backscatter, $S_{c b}(x, y)$, is then computed as ${ }^{1}$

$$
\begin{aligned}
S_{c b}(x, y) & =\frac{D_{f}(x, y)}{D_{f}(x, y)+D_{b}(x, y)} / \frac{D_{f}\left(x_{0}, y_{0}\right)}{D_{f}\left(x_{0}, y_{0}\right)+D_{b}\left(x_{0}, y_{0}\right)} \\
& =\left[1+R\left(x_{0}, y_{0}\right)\right] /[1+R(x, y)],
\end{aligned}
$$

in which the reference field has a jaw setting of $\left(x_{0}, y_{0}\right)$, most commonly a $10 \mathrm{~cm} \times 10 \mathrm{~cm}$ symmetric field. The $S_{c b}(x, y)$ was then used to correct for the effect of the backscatter on the total photon output, $S_{c p}(x, y)$, which is a product of the head scatter factor $S_{c}(x, y)$, the phantom scatter factor $S_{p}(x, y)$, and the backscatter correction $S_{c b}(x, y)$. The first two factors have been computed for photon fields using a convolution/superposition dose calculation algorithm ${ }^{1}$ as described in a previous work.

To validate the above "sum-of-the-components" method in computing the backscatter, we compared the calculated $R(x, y)$ to the results from the direct Monte Carlo simulations of various jaw settings. the $S_{c b}(x, y)$ obtained from the Monte Carlo simulation was also compared to the measured data based on counting the electronic feedback pulses or accumulative charge from the electron target. ${ }^{1,11}$ To evaluate

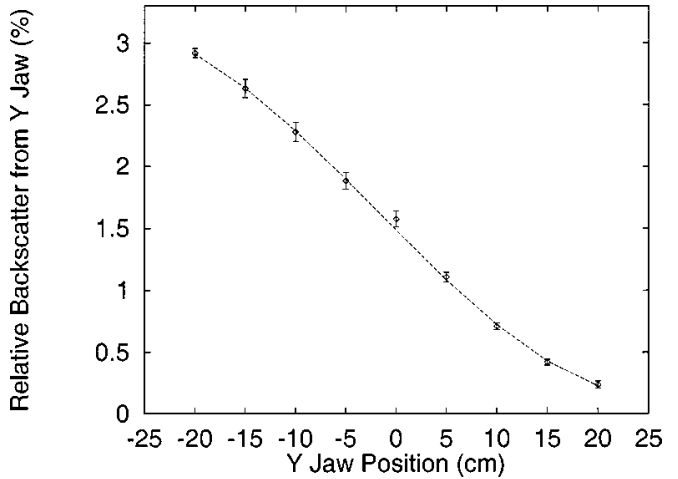

FIG. 2. Relative backscatter from the $Y_{1}$ jaws as a function of the jaw position $R_{y_{1}}\left(y_{1}\right)$. The $Y_{2}$ jaw is parked at $20 \mathrm{~cm}$ off axis. The one standard error of the data is shown by the error bar. The data were fit to a polynomial function as shown by the dotted line.

the effect of the backscatter on treatment fields using jaw movement, $S_{c p}(x, y)$ was also calculated for treatment fields using dynamic wedges.

\section{RESULTS AND DISCUSSION}

Results of the relative backscatter from the $Y_{1}$ jaw as a function of the jaw position, $R_{y_{1}}\left(y_{1}\right)$, are shown in Fig. 2 . The negative index of the $Y_{1}$ jaw position means that the $Y_{1}$ jaw passes the central axis and approaches the parked $Y_{2}$ jaw. These data show that the backscattered radiation increases with a smaller $Y$ jaw opening. The maximum dose from the backscattered radiation is about $3 \%$ compared with the dose from the forward radiation. The standard error of $R_{y_{1}}\left(y_{1}\right)$ is $3 \%-5 \%$ of the values shown in Fig. 2. To interpolate $R_{y_{1}}\left(y_{1}\right)$ for any $y_{1}$ position, data of Fig. 2 were fit to a three-order polynomial function of

$$
\begin{aligned}
R_{y}(y)= & \left(1.54 \pm 2.02 \times 10^{-2}\right)+\left(-8.45 \times 10^{-2} \pm 3.80\right. \\
& \left.\times 10^{-3}\right) y+\left(4.47 \times 10^{-5} \pm 1.07 \times 10^{-5}\right) y^{3} .
\end{aligned}
$$

This function is chosen because the collimator jaw and the monitor chamber are symmetric against the central axis of the accelerator. Results of the $R_{y_{1}}$ show a small degree of nonlinearity because the increase of the $R_{y_{1}}$ with $y_{1}$, or the gradient of $R_{y_{1}}$, is greater near the central axis than at off axis. The sigmoidal shape of the $R_{y_{1}}$ function suggests that the $Y$ jaw backscatter may follow a bell-shaped distribution because the backscattered near the central axis is more likely to reach the monitor chamber.

Results of the relative backscatter from the $X_{1}$ jaw with the fully opened $Y$ jaws, $R_{x_{1}}\left(x_{1}, y_{1}=y_{2}=20\right)$, are shown in Fig. 3. These data indicate that because the $X$ jaws are further away from the monitor chamber, the maximum backscatter from the $X$ jaws is only $0.8 \%$ of the forward radiation. The standard error of $R_{x_{1}}\left(x_{1}\right)$ ranges within 5\%-10\% of the values shown in Fig. 3. These data were also fit to a linear function of 


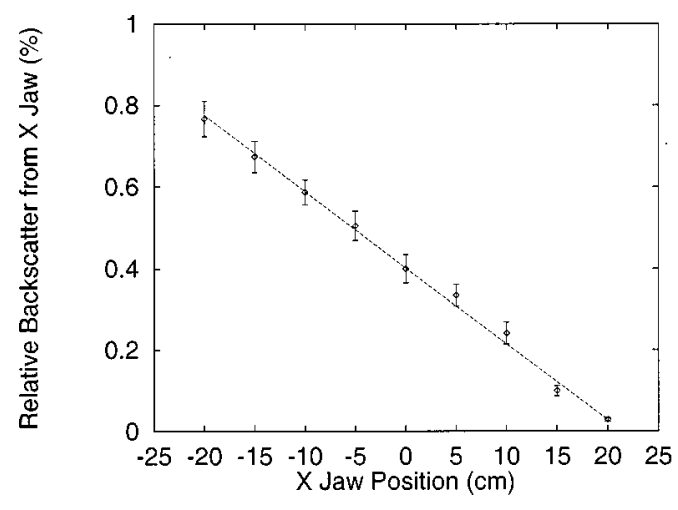

FIG. 3. Relative backscatter from the $X_{1}$ jaws as a function of the jaw position, $R_{x_{1}}\left(x_{1}, y_{1}=y_{2}=20\right)$. The $X_{2}$ jaw is parked at $20 \mathrm{~cm}$ off axis. The $Y$ jaws are completely opened. The one standard error of the data is shown by the error bar. The data were fit to a polynomial function as shown by the dotted line.

$$
\begin{aligned}
R_{x}(x)= & \left(0.40 \pm 8.53 \times 10^{-3}\right) \\
& +\left(-1.87 \times 10^{-2} \pm 4.55 \times 10^{-4}\right) x .
\end{aligned}
$$

Figure 3 shows that the $X$ jaw backscatter more closely follows a linear function than the $Y$ jaw backscatter. This may be because the $X$ jaws are located further away from the monitor chamber. Thus, both the magnitude and directional preference of the backscatter are greatly reduced. In a sense, the $X$ jaw backscatter is more likely to be proportional to the area that the $X$ jaws are exposed to the monitor chamber. As a result, $P_{y}\left(y_{1}, y_{2}\right)$ obtained with the maximum $X$ jaw backscatter (see the following data) was applied to other fields with different $X$ jaw openings.

Results of the backscatter from the fully closed $X$ jaws as a function of the $Y_{1}$ jaw position are shown in Fig. 4. The effect of the $Y$ jaw blocking on the $X$ jaw backscatter is clearly demonstrated. The corresponding $P_{y_{1}}\left(y_{1}\right)$ is shown in Fig. 5. Because $P_{y_{1}}\left(y_{1}\right)$ should be symmetric against the central axis, and passes zero at the central axis, as discussed in Sec. II, we fit the data of $P_{y_{1}}\left(y_{1}\right)$ to a three-order polynomial function of

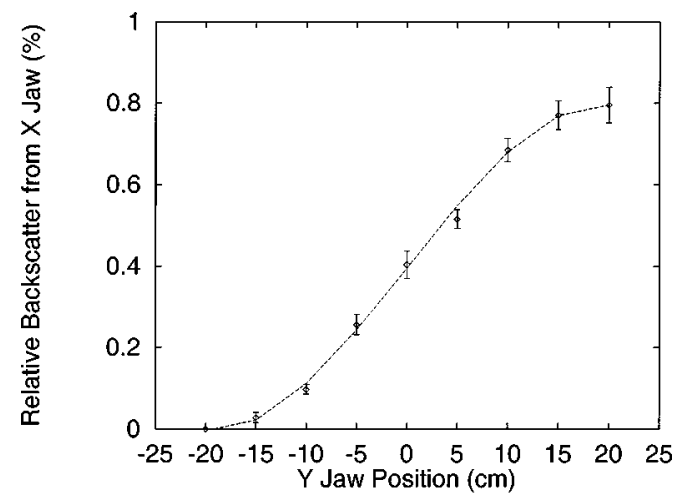

FIG. 4. Relative backscatter from the $X$ jaws as a function of the $Y_{1}$ jaw position. The $X$ jaws are completely closed to allow the maximum backscatter. The $Y_{2}$ jaw is parked at $20 \mathrm{~cm}$ off axis. The one standard errors of the data is shown by the error bar.

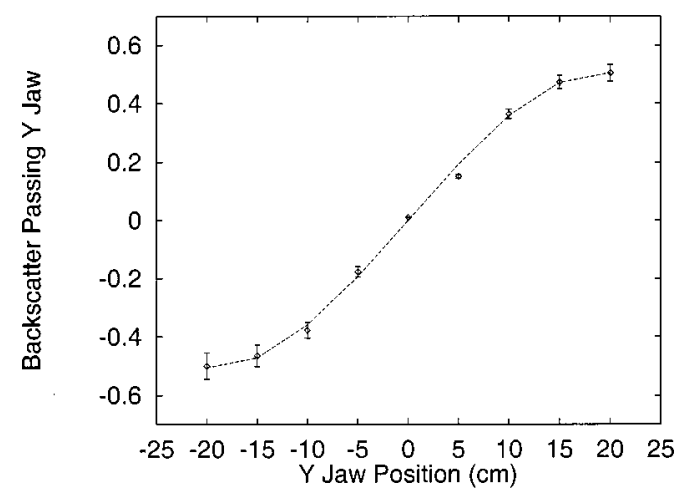

FIG. 5. The relative amount of the $X$ jaw backscatter that passes the $Y_{1}$ jaw as a function of the jaw position, $P_{y_{1}}\left(y_{1}\right)$. The $X$ jaws are completely closed to allow the maximum backscatter. The $Y_{2}$ jaw is parked at $20 \mathrm{~cm}$ off axis. The one standard error of the data are shown by the error bars. The data were fit to a polynomial function as shown by the dotted line.

$$
\begin{aligned}
P_{y}(y)= & \left(3.95 \times 10^{-2} \pm 9.92 \times 10^{-4}\right) y \\
& +\left(-3.55 \times 10^{-5} \pm 2.25 \times 10^{-6}\right) y^{3} .
\end{aligned}
$$

The results of $P_{y_{1}}\left(y_{1}\right)$ also exhibit a sigmoidal shape, which suggests that the passing of the $X$ jaw backscatter is greater when the $Y$ jaw opening is located near the central axis. This is because the backscatter going through such an opening is more likely to reach the monitor chamber. We have also compared $P_{y_{1}}\left(y_{1}\right)$ of the fully closed $X$ jaws with that of an $X$ jaw opening of $20 \mathrm{~cm}$. Results showed that the change of $P_{y_{1}}\left(y_{1}\right)$ with the $X$ jaw opening is within two standard deviation of $P_{y_{1}}\left(y_{1}\right)$. This supports the assumption that the $P_{y_{1}}\left(y_{1}\right)$ obtained with the fully closed $X$ jaws can be applied to fields with a different $X$ jaw opening.

Using data from Figs. 2-5, the total backscatter from the jaws was computed using Eqs. (2)-(5). Take an example of an asymmetric field of $10 \mathrm{~cm} \times 10 \mathrm{~cm}$ with $X_{1}$ and $Y_{1}$ jaws at the central axis, $X_{2}$ and $Y_{2}$ jaws at $10 \mathrm{~cm}$ off axis. Our calculation shows that

$$
\begin{aligned}
& R_{y_{1}}\left(y_{1}\right)=(1.54 \pm 0.02) \%, \\
& R_{y_{2}}\left(y_{2}\right)=(0.74 \pm 0.01) \%, \\
& R_{x_{1}}\left(x_{1}, y_{1}=y_{2}=20\right)=(0.40 \pm 0.01) \%, \\
& R_{x_{2}}\left(x_{2}, y_{1}=y_{2}=20\right)=(0.21 \pm 0.00) \%, \\
& P_{y_{1}}\left(y_{1}\right)=0.00 \pm 0.00, \\
& P_{y_{2}}\left(y_{2}\right)=0.36 \pm 0.00 .
\end{aligned}
$$

Thus,

$$
\begin{aligned}
& R_{y}\left(y_{1}, y_{2}\right)=(2.28 \pm 0.02) \%, \\
& R_{x}\left(x_{1}, x_{2}, y_{1}, y_{2}\right)=(0.22 \pm 0.01) \%, \\
& R(x, y)=(2.50 \pm 0.02) \% .
\end{aligned}
$$

This example shows that the backscatter from the $X$ jaw is approximately $10 \%$ of that from the $Y$ jaws. In general, this 


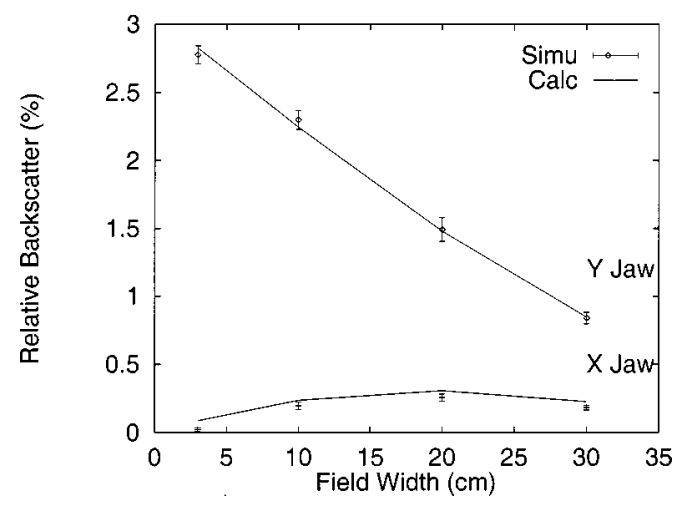

FIG. 6. Relative backscatter from the $X$ and $Y$ jaws, $R_{x}$ and $R_{y}$, as a function of the jaw opening. The discrete points ("simu") show results from the direct Monte Carlo simulations of these fields; the continuous lines ("calc") show the data from the model-based calculations. The one standard errors of the data are shown by the error bars.

holds true for most of the fields we have studied. As a result, the total backscatter can be approximated primarily by that from the $Y$ jaws.

To verify the above calculation, we compared the $R_{y}$ and $R_{x}$ with those from the direct Monte Carlo simulations of the same fields. Figure 6 shows this comparison for symmetric fields of $3 \mathrm{~cm} \times 3 \mathrm{~cm}$ to $30 \mathrm{~cm} \times 30 \mathrm{~cm}$. The results indicate that the calculated $R(y)$ agree with those from the direct simulations within one standard error. The $R(x)$ calculation slightly overestimates its simulated value particularly for the $3 \mathrm{~cm} \times 3 \mathrm{~cm}$ field. We believe that with this small $Y$ jaw opening, particles from the $X$ jaws are more likely to be scattered between the opposing $Y$ jaws rather than to be scattered back to the monitor chamber. As a result, $P_{y_{1}}$ and $P_{y_{2}}$ become correlated, which causes $P_{y}$ calculated by the sum of $P_{y_{1}}$ and $P_{y_{2}}$ to be overestimated. Fortunately, because the magnitude of $R_{x}$ is insignificant compared to $R_{y}$ for the small field, the overestimation of $P_{y}$ does not have a strong effect on the total backscatter.

To further evaluate the calculation model, the $X$ and $Y$ jaw backscatter were also determined for asymmetric fields from the calculations and direct simulations. Figure 7 shows the comparison for $10 \mathrm{~cm} \times 10 \mathrm{~cm}$ fields with the center of the fields located at the central axis, and off-axis locations of 5, 10 , and $15 \mathrm{~cm}$. For example, with the center of the field at 10 $\mathrm{cm}$ off axis, the $X 1, X 2, Y 1$, and $Y 2$ are located at 10, 0, 10, $0 \mathrm{~cm}$, respectively. The results in Fig. 7 show that the $R_{y}$ calculations for these asymmetric fields agrees with the simulation within one standard error except for the last field. The calculation seems to predict a higher degree of increase with the off-axis location of the field than the simulation. This may be caused by the statistical uncertainty in fitting the $R_{y_{1}}$ data [Eq. (14)] to the third-order polynomial function. In a sense, the more $R_{y_{1}}$ approaches linear, the less that $R_{y}$ will increase with the off-axis location. Thus, the parameter of the $y^{3}$ term directly determines how the $R_{y_{1}}$ changes with off-axis locations. Figure 7 also shows that the calculated $R_{x}$ is greater than the simulated results by a small amount, which is believed to be caused by the overestimation of $P_{y}$

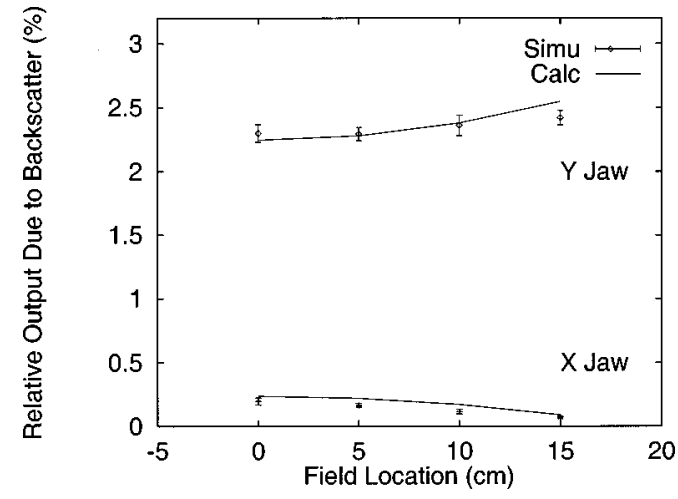

FIG. 7. Relative backscatter from the $X$ and $Y$ jaws, $R_{x}$ and $R_{y}$, for a $10 \mathrm{~cm} \times 10 \mathrm{~cm}$ field at different off-axis locations. The discrete points ("simu") show results from the direct Monte Carlo simulations of these fields; the continuous lines ("calc") show the data from the model-based calculations. The one standard errors of the data are shown by the error bars.

as indicated previously. Opposite to the $Y$ jaw backscatter, the $X$ jaw backscatter decreases slightly with the off-axis location because the $Y$ jaw passing $P_{y}$ decreases with an asymmetric field, as discussed earlier for results in Fig. 4. As a result, the total backscatter as the sum of $R_{y}$ and $R_{x}$ is not strongly dependent on the asymmetry of the field. In other words, the width of the jaw opening rather than its location determines the magnitude of the total backscatter.

To evaluate the Monte Carlo calculated results against measurement, we computed $S_{c b}$ using Eq. (12) and compared with those from counting the electron target pulses or charge. ${ }^{1,11}$ Figure 8, shows $S_{c b}$ as functions of the jaw openings from our calculation and from the measurement of Lam, Muthuswarmy, and Haken. ${ }^{11}$ Data of " $Y$ jaw" are $S_{c b}$ for different $Y$ jaw openings with the $X$ jaws completely opened. The $Y$ jaw opening of $40 \mathrm{~cm}$ is used as the reference field to compute $S_{c b}$. Similarly, data of " $X$ jaw" are $S_{c b}$ for different $X$ jaw openings with the $Y$ jaws completely opened. The standard error of the measured data is approximately $0.2 \%$ of its absolute value. Figure 9 also shows the comparison of $S_{c b}$ from the Monte Carlo data and from measurement of elec-

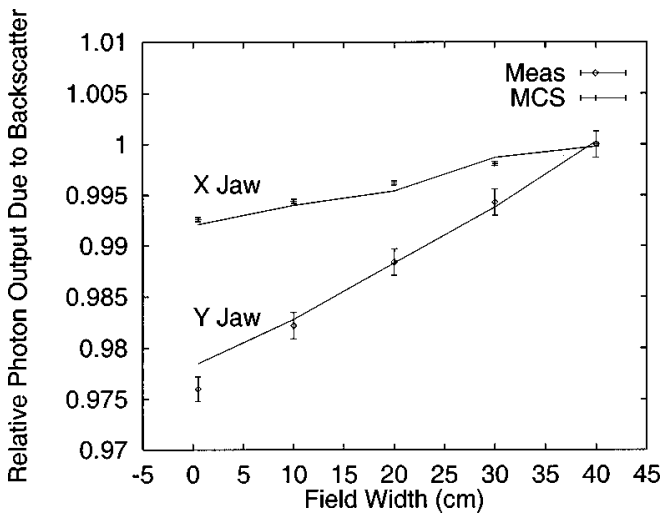

FIG. 8. Relative photon output due to the backscatter, $S_{c b}$, as a function of the $X$ jaw and $Y$ jaw openings with the other jaw pairs completely opened. The straight line ("meas") shows the data from measurement of electron target charge. The discrete points ("MCS") are from the Monte Carlo simulation. The one standard errors of the data are shown by the error bars. 


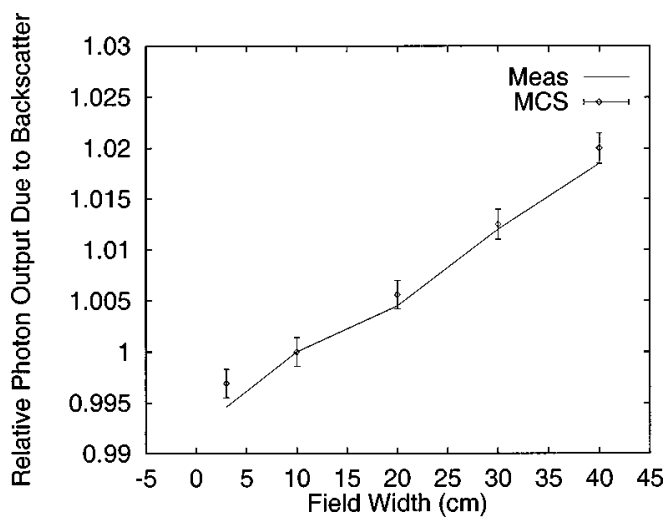

FIG. 9. Relative photon output due to the backscatter, $S_{c b}$, of symmetric and square fields. The straight line ("'meas") shows data from measurement of electron target pulses. The discrete points ("MCS") are from the Monte Carlo simulation. The one standard errors of the data are shown by the error bars.

tron target pulses $^{1}$ for a series of symmetric fields from $3 \mathrm{~cm} \times 3 \mathrm{~cm}$ to $40 \mathrm{~cm} \times 40 \mathrm{~cm}$. The $10 \mathrm{~cm} \times 10 \mathrm{~cm}$ field was used as the reference field in computing the relative photon output. The standard error of the measured data is approximately $0.4 \%$ of its absolute value.

The above-mentioned results indicate that the change of the photon output due to the backscatter is predicted to within $0.5 \%$ of the values of $S_{c b}$ by the Monte Carlo based data compared to the measurement. This accuracy is clinically acceptable in computing photon output for monitor units. The discrepancy seems to apply only to the smaller field less than $10 \mathrm{~cm}$ wide, which might be due to the uncertainty in the measured data, because results from the two measurements differed from each other. The increase of the photon output is the order of $2 \%$ from the $10 \mathrm{~cm}$ field to the $40 \mathrm{~cm}$ field, which is mostly caused by the $Y$ jaw backscatter, as shown in Fig. 8. This also agrees very well with recently published results for Varian machines. ${ }^{5,11}$

We have computed the relative backscatter for dynamic wedges. The results of $S_{c b}$ are shown in Fig. 10 for these fields. The results show that for dynamic wedges using the upper jaw movement, the photon output due to the backscatter increases when the jaw is closing. This will cause a small

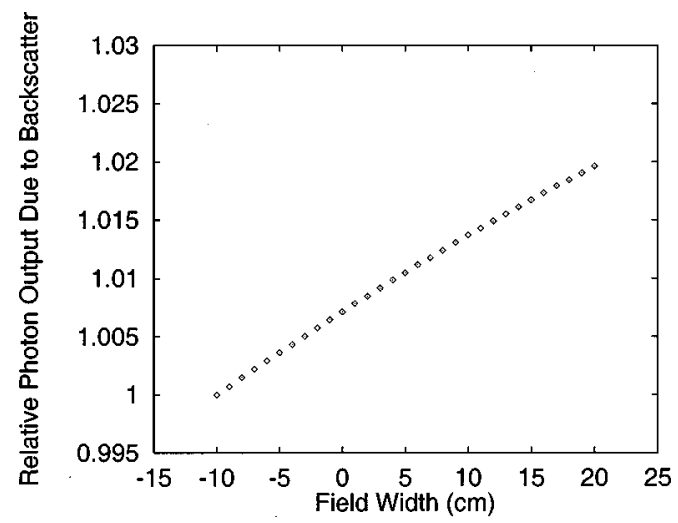

FIG. 10. Relative photon output due to the backscatter, $S_{c b}$, for dynamicwedged fields with moving $Y$ jaws. deviation from the intended fluence modulation specified by the segmented treatment table. Therefore, the effect of the backscatter may need to be taken into account for a more accurate dose calculation. On the other hand, if the dynamic wedge uses the $X$ jaw motion instead of the $Y$ jaw motion to create the wedged profile, the effect of the backscatter will be minimal. For fields with additional blocks or multileaf collimators (MLC), the backscatter from these components is negligible because they are even further away from the monitor chamber. Thus, the jaw settings for these blocked fields can be used directly to determine the backscattered radiation. If the jaws move along with MLC edges to reduce the leaf leakage, the effect of the jaw backscatter may need to be considered when designing fluence modulation for intensity-modulated fields.

We have observed that the effect of the backscatter is more significant on monitor chambers with entrance and exit windows made of kapton than those made of mica. The chambers with mica windows exhibited very little dependence of the monitor-chamber-scored dose on the jaw opening. This is because the backscattered radiation is mostly composed of low-energy photons and electrons, which are very easy to eliminate from the beam. For example, Hounsell showed that an antibackscatter plate was effective in reducing the backscattered radiation to the monitor chamber for intensity-modulated fields. Therefore, a different construction of the monitor chamber might be considered to minimize the effect of the backscattered radiation and to simplify the calculations of monitor units, particularly for treatment fields using dynamic jaw motions.

\section{SUMMARY}

A Monte Carlo technique was used to simulate clinical accelerators and to study the effects of the backscattered radiation from collimator jaws to monitor chambers. Based on the results of the Monte Carlo simulations, dose scored within the monitor chamber was separated into those from the forward and from the backscattered radiation. The backscattered dose was further modeled as functions of the $X$ and $Y$ jaw positions. Therefore, the total backscatter for any jaw setting was calculated as a sum of those components from the individual jaws. Our results show that the $Y$ jaws, which are closer to the monitor chamber, contribute to the majority of the backscattered radiation. The amount of the backscatter from the $X$ jaws is about $10 \%$ of that from the $Y$ jaws. The backscatter decreases approximately in a linear fashion with the jaw opening, which results in an increase of photon output by about $2 \%$ from a $10-\mathrm{cm}$-wide field to a $40-\mathrm{cm}$-wide field. This conclusion agrees well with previous published data for Varian 2100C accelerators. The width of the jaw opening rather than its off-axis position has greater influence on the change of the photon output due to the backscatter. This study shows that to model the photon output accurately in computing monitor units, it is necessary to account for the effect of the backscatter from the collimator jaws, particularly for machines using kapton chambers located close to the upper collimator jaws. 


\section{ACKNOWLEDGMENTS}

The authors would like to thank Dr. Dave Rogers at the National Research Council of Canada for providing the BEAM Monte Carlo code and Dr. Kwok Lam at the University of Michgan for comparing to their measurement data. We also appreciate communications on Monte Carlo techniques with Dr. Frank Verhaegen at the Royal Marsden Hospital and his review of the manuscript.

${ }^{a)}$ Electronic mail: hliu@mdanderson.org

${ }^{1}$ H. H. Liu, T. R. Mackie, and E. C. McCullough, "Calculating output factors for clinical photon beam radiotherapy using a convolution/ superposition method based on a dual source photon beam model," Med. Phys. 24, 1975-1985 (1997).

${ }^{2}$ H. H. Liu, T. R. Mackie, and E. C. McCullough, “A dual source photon beam model used in convolution/superposition dose calculations for clinical megavoltage x-ray beams," Med. Phys. 24, 1960-1974 (1997).

${ }^{3} \mathrm{H}$. Kubo, "Telescopic measurements of backscattered radiation from secondary collimator jaws to a beam monitor chamber using a pair of slits," Med. Phys. 16, 295-298 (1989).

${ }^{4}$ C. Duzenli, B. McClean, and C. Field, "Backscatter into the beam monitor chamber: Implications for dosimetry of asymmetric collimators," Med. Phys. 20, 363-383 (1993).

${ }^{5}$ M. K. Yu, R. S. Sloboda, and F. Mansour, "Measurement of photon beam backscatter from collimators to the beam monitor chamber using target-current-pulse-counting and telescope techniques," Phys. Med. Biol. 41, 1107-1117 (1996).

${ }^{6}$ K. L. Lam, M. S. Muthuswarmy, and R. K. T. Haken, "Flattening-filterbased empirical methods to parametrize the head scatter factor," Med. Phys. 23, 343-352 (1996).
${ }^{7}$ A. R. Hounsell, "Monitor chamber backscatter for intensity modulated radiation therapy using multileaf collimators," Phys. Med. Biol. 43, 445-454 (1998).

${ }^{8}$ P. Huang, J. Chu, and B. E. Bjärngard, "The effect of collimator backscatter radiation on photon output of linear accelerators," Med. Phys. 14, 268-269 (1987).

${ }^{9}$ D. L. Watts and G. S. Ibbott, "Measurement of beam current and evaluation of scatter production in an $18-\mathrm{MeV}$ accelerator," Med. Phys. 14, 662-664 (1987).

${ }^{10}$ M. B. Sharpe, D. A. Jaffray, J. J. Battista, and P. Munro, “Extrafocal radiation: A unified approach to the prediction of beam penumbra and output factors for megavoltage x-ray beams," Med. Phys. 22, 2065-2074 (1995).

${ }^{11}$ K. L. Lam, M. S. Muthuswarmy, and R. K. T. Haken, "Measurement of backscatter to the monitor chamber of medical accelerators using target charge," Med. Phys. 25, 334-338 (1998).

${ }^{12}$ T. R. Mackie, H. H. Liu, and E. C. McCullough, "Model based photon dose calculation algorithms," in Treatment Planning in Radiation Oncol$o g y$, edited by F. M. Khan and R. A. Potish (Williams and Wilkins, Baltimore, MD, 1998), pp. 89-112.

${ }^{13}$ D. W. O. Rogers, B. A. Faddegon, G. X. Ding, C. M. Ma, J. We, and T. R. Mackie, "BEAM: A Monte Carlo code to simulate radiotherapy treatment units," Med. Phys. 22, 503-524 (1995).

${ }^{14}$ C. Ma, B. A. Faddegon, D. W. O. Rogers, and T. R. Mackie, " Accurate characterization of Monte Carlo calculated electron beams for radiotherapy," Med. Phys. 24, 401-416 (1997).

${ }^{15}$ E. L. Chaney and T. J. Cullip, "A Monte Carlo study of accelerator head scatter,' Med. Phys. 21, 1383-1390 (1994).

${ }^{16}$ D. M. J. Lovelock, C. S. Chui, and R. Mohan, "A Monte Carlo model of photon beams used in radiation therapy," Med. Phys. 22, 1387-1394 (1995). 